\title{
Localization in Wireless Sensor Networks for Accurate Event Detection
}

\author{
Amit Sharma, JUIT Wakhnaghat, India \\ Pradeep Kumar Singh, ABES Engineering College, Ghaziabad, India \\ iD https://orcid.org/0000-0002-7676-9014
}

\begin{abstract}
Event detection at its initial stage is considerably most demanding and more importantly challenging to reduce the causes and damages. The GPS-enabled sensor nodes are possibly a solution for the location estimation, but having GPS receiver in each sensor node makes the network costly. In this paper, the authors have presented a UNL, unknown node localization, method for the estimation of sensor location. The proposed method is based on RSSI, and there is no requirement of extra hardware and communication of data among the sensor nodes. The experiments are conducted in order to investigate the localization accuracy of UNL method, and they analyzed that the proposed method is simple as there is less computation and communication overhead. The proposed algorithm is further compared with other existing localization methods for the accurate estimation of unknown nodes. The experimental results show the effectiveness of the algorithm and its capability for locating the unknown nodes in a network more accurately.
\end{abstract}

\section{KEYWORDS}

Anchor Nodes, Node Localization, Receive Signal Strength Indicator, Unknown Node, Wireless Sensor Networks

\section{INTRODUCTION}

In recent times for the medical implantations of sensor the wireless communication devices are studied extensively. For healthcare, several of studies and various implantable devices like wireless body sensors are gaining attention. The implantable devices can perform the real time detection of any problem and present condition of body that can be accessed from anywhere at any time from medical personnel of by any individual. Wireless Sensor Networks serves variety of applications such as healthcare, defense, industrial, environmental and many others. The objective of this study is to present an algorithm for the localization of sensor nodes that triggers the event. Critical events such as fires can occur across different environments such as forests, commercial, residential, and/or even open spaces. While newer techniques have evolved to detect and provide alerts on fire emergencies, early detection methods relied mostly on humans. Today, human-based observations have been replaced by Satellite-based systems. Yet, as satellite-based observations are not continuous with time (Marta et al., 2009), these systems are nonetheless not suited for the early detection of fire but more likely for the purpose of monitoring and alerts, covering areas of any size. 
With recent advances in technology, Wireless Sensor Networks (WSNs) have gained attention from researchers. Here, the development of various sensors makes it much easier to gather the correct information parameters from the environment. With increasing number of devices that may be added to cover an area of any size, a key advantage of WSNs is their scalability. Recent sensor technological innovations have aided in the gathering of various environmental parameters in real time. Sensors works collaboratively for the detection of a critical event; in the case of wildfire, it predicts well before the risk of such a disaster emergency so that first respondents may be dispatched to event locations on time (Kim \& Han, 2015).

As noted, fires can occur in open spaces, forests and residential areas. One of the easiest ways to detect fire is the use of smoke detectors. Other means include the use of sensors, which are responsive towards ionization and obscuration. A problem with these detectors is the generation of false alarm via similar color of fire regions, for example, the smoke from toasting bread and/or cigarette smoking. Even so, the advantages of WSNs towards critical event detection and the periodic collection of data made it suitable for manufacturing applications. The periodical collection of data is essential for operations such as the tracking of critical events and the monitoring of health status, reducing costly human labor and errors (Shahzad et al., 2016). In WSNs, the localization can determine the physical coordinates of a number of sensor nodes in the network.

The rest of this paper is organized as follows. In Section 2, we review the background vis-à-vis the extant literature on localization, specifically for WSNs. In Section 3, a localization algorithm for the computation of position and distance among the sensor nodes within the context of WSNs is advanced. Following this, we discuss the implementation of the proposed system in Section 4. In Section 5, several cases are experimented for the analysis of the algorithm. We then provide concluding remarks and insight into future work in Section 6.

\section{BACKGROUND}

Localization may be conceived as the process to determine the locations of devices connected within a network, such as a WSN. WSN comprises a large number of sensor nodes that are deployed in an area of interest for the measurement of any phenomenon (Maddumabandara et al., 2015). The prime objective of localization is to compute the target location. The use of global positioning system (GPS) is not realistic in this application context; therefore, sensors are required to self-organize a coordinate system. Localization is the estimated unknown node position through communication between localized nodes and un-localized nodes. The routing services heavily rely on the location information of the sensor nodes.

A sensor network localization algorithm (Han et al., 2016; Patwari et al., 2005) typically entails three main phases:

1. Distance Estimation

2. Computation of Position

3. Localization Algorithm

In the distance estimation phase, the relative distance among the sensor nodes is estimated via measurement techniques. Coordinates of the unknown nodes are computed in the position computation phase with respect to the anchor node and other neighboring sensor nodes. The final phase, the localization algorithm, generally determines how the information related to the position and distance is manipulated to allow most or every node in WSN to estimate their own position (Khan et al., 2017). Additionally, localization algorithm may embed other algorithms for the reduction of errors and to refine the position of nodes. The distance is estimated by using four most common methods, including 
the angle of arrival (AoA), the time of arrival (ToA), the time difference of arrival (TDoA), and the received signal strength indicator (RSSI).

AoA is a technique for the distance estimation in which each sensor evaluates the relative angles among the received radio signals. In ToA method, the measures that are based on time are used to estimate the distance between two nodes. TDoA is a technique used to determine the distance among the mobile station and its nearest base station. RSSI methods are applied to translate received signal strength into distance, most commonly for computing lateration and angulation positions. The lateration positioning computation depends on the accurate measurements of three non-collinear anchor nodes. Multi-lateration refers to the lateration that consists of more than three anchors. In contrast, the computation of position via angulation or triangulation depends on the knowledge of angles in place of distance (Gu et al., 2013).

In the literature, various localization techniques for both indoor and outdoor environments have been studied. Superior techniques that perform better in terms of less energy efficiency, higher accuracy and less shadowing effects exist. Here, the proposed algorithm is capable of estimating the position of unknown node via less connectivity and information of proximity in network. The algorithm also provides higher level of accuracy for estimated positions.

\subsection{Classification of Localization Methods}

On the basis of sensor implementation, localization algorithms for WSN are classified into four categories:

1. Centralized vs. distributed

2. Anchor-based vs. anchor-free

3. Range based vs. range free

4. Stationary vs. mobile

\subsubsection{Centralized v. Distributed}

In the centralized method, each of the computation is achieved at the central server. Data collected from the complete network are transmitted to the central server, which further compute the estimated location of each sensor node in a WSN. In the distributed method, the computation is distributed among the nodes. Each of the sensor nodes is responsible for estimating its own position vis-à-vis the information collected from its neighbors (Shi et al., 2014).

\subsubsection{Anchor-Based v. Anchor-Free}

Anchor nodes are nodes that know their coordinates via the use of GPS or manual placement. In anchor-based techniques, the anchor nodes have GPS whereas all other nodes compute their location via trilateration. The anchor-free methods are used for connectivity only with the location of the nodes specifying the position of the sensor node being relative to each other (Khan et al., 2009).

\subsubsection{Range-Based v. Range-Free}

The range-based method utilizes the calculated angle and distance to estimate location via the principles of geometry. In contrast, range-free method utilizes the pattern matching and connectivity technique to estimate location. Here, the distances are not calculated directly but the hop counts are used. After the hop counts are determined, the distance among the nodes are estimated via an average distance and then the principles of geometry are used for the computation of location (Alasiry \& Ohyama, 2012).

\subsubsection{Stationary v. Mobile}

The design of localization algorithms depends on the sensor field where they are deployed. The nature of some sensor nodes is static and thus fixed at one place. Most applications use these static 
nodes for the purpose of monitoring. In few WSN applications, sensor nodes are mobile in which the nodes are more versatile (then static ones) and can be deployed in any situation for coping with any environmental changes (Yoon et al., 2011).

\subsection{Localization Related Work}

In this section, popular existing localization methods are highlighted. The localization methods may be classified as range-based v. range-free. In range-based algorithms, the distance seems to be estimated first prior to computing a position. The distance estimation can be done via different ranging techniques, including RSSI, TOA and AoA. Afterwards, the position is computed via triangulation, trilateration and multi-lateration (Ali et al., 2010; Zhang and $\mathrm{Yu}, 2008$ ). In range-free algorithms no ranging technique is needed; instead, the position is estimated based on network connection information rather than the estimated distance or angles. Typical examples of range-free techniques include ad hoc positioning system or APS and approximate point in the triangulation or APIT (Wan et al., 2018; Yuan et al., 2018).

The process of localization entails gauging the physical coordinates of a number of static WSNs (Patwari et al., 2005). In the last few years, researchers have paid attention to the localization of wireless sensor nodes in WSN. This is because in a disaster emergency, there occurs the loss of habitants, death and harm to individuals, even the complete ruining of places. Hence, it is very important to find out individuals that may be affected and to accurately locate the spots at any cost. In some cases, more than two kinds of physical measurements are executed in parallel via a few of the positioning systems; this way, more information about the target node can be obtained and positioning accuracy and robustness amplified. The time of arrival, the angle of arrival, the time difference of arrival and received signal strength indicator are examples of these multimodal localization schemes. The two vital issues that occur in localization are the directional framework characterization and the separation uncertainty among the sensors. These issues may be resolved via centralized vs. distributed, anchorfree vs. anchor-based and range-free vs. range-based methodologies (Gu et al., 2013).

There are various localization algorithms designed for the distance estimation. Yet, no single algorithm may be superior for use in the entire WSN applications. As an example, the algorithm that is suited for mobile sensor nodes may not work efficiently with the static sensor nodes. A framework can only be designed by combining all of the techniques that can serve for the different applications. Recent research on localization algorithms in WSNs has appeared in (Luan et al., 2014; Cao, 2011; Bianchi et al., 2018). Here, we highlight the more popular localization techniques and range-free algorithms of localization, including the mobile reference nodes. Some sensor nodes in a WSN are generally equipped with GPS, which enable them to know their own locations (Haque \& Assi, 2015). Nodes with known positions are classified as reference or anchor nodes. In contrast, nodes initially with unknown location are classified as unknown sensor nodes. Any sensor nodes in a network is capable of estimating its location by implementing range-free or range-based methods when there exists three or more than three anchor nodes in its two-dimensional coverage field. The accuracy of the localization is highly influenced by the number and the position of anchor nodes. Recent studies show that the accuracy of localization increases with an increasing number of anchor nodes (Zhao et al., 2018).

The main issue in increasing the number of anchor nodes is that their cost is high in comparison with the rest of the normal sensors. In a simple network, if ten percent of the sensors nodes are anchor nodes, then the cost of the network is ten times higher than its normal network. It is also purported that once the position coordinates of the unknown nodes is localized, the anchor nodes becomes ineffective. This is the basis for specifying a single anchor node for the localization of sensor network. Similar techniques use a common feature called range-based approaches. Even so, these techniques can get efficient results but either the cost for the hardware is expensive or their results can be determined by signal propagation assumptions that are unrealistic. The alternate and a cost-effective solution due to the limitations of hardware in sensor devices are range-free approaches. (Chen et al., 2011) 
have proposed an algorithm for the computation of location by considering a centroid among its approximate anchor nodes. DV Hop provides a solution that offers multiple-hop flooding instead of single-hop broadcast. Thus, the normal sensor nodes are capable of finding their distance from the anchor nodes via hop counts. A similar approach to DV Hop is adopted by (Zeng et al., 2018) which is a positioning technique. The main difference between range based and range free schemes is that the technique of amorphous positioning provides much improvement over the estimation of location by utilizing the estimation of offline hop distance during the exchange of information among its neighbors. (Ma et al., 2012) have proposed an algorithm based on range-free approaches called as APIT algorithm, which solves the problem of localization by dividing the area to triangular region among anchor nodes. A test names point in the triangle is conducted for the estimation of location through triangles, which are formed by anchor nodes. Hence, this test decreases the probable residing area in which the node exists. On the basis of this survey, localization via single mobile anchor node is more economical. Additionally, by considering the limitations of computation and less memory power of sensor nodes (Stojkoska et al., 2009; Phoemphon et al., 2018), we adopted a method that uses mobile anchor nodes for locating sensors in a WSN.

\section{THE PROPOSED ALGORITHM}

Figure 1 is a flow diagram of the localization process.

In this process, a localization algorithm for computing the location of sensor nodes is proposed. Each of the regular nodes in a network using this algorithm estimates its distance only to the reliable nodes. The system of $\mathrm{n}$ wireless sensor nodes is uniformly distributed in a square area. All nodes are assumed to have same transmission capability and each node is able to communicate directly with any other node.

In the first step, any anchor-based localization algorithm locates anchor and broadcasts message via the network where $\mathrm{N}$ is the hop count value initialized to 1 . When a node receives a message, it

Figure 1. Flow Diagram of the Localization Algorithm

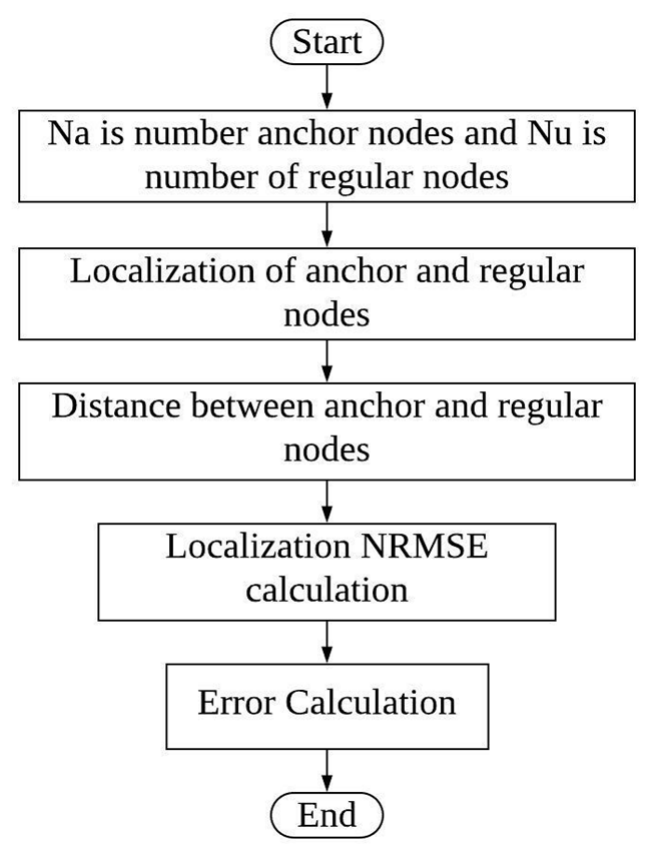


stores the gate anchor position as well as the receiver hop count in its database and adds 1 to the hop count value and broadcasts the resulting message. When this message is received by another node, it checks its database information. If the anchor information exists in the database and the received hop count value $\mathrm{N}$ is smaller than the stored one, then the node updates and broadcasts the resulting message. If the stored value is smaller than $\mathrm{N}$, then the node discards the received message.

At the receiving end, the gate anchor becomes aware of its own position as well as all of those other anchor nodes in the network. The anchor also computes the estimate of the distance to any other anchor and corresponding estimation error. The proposed algorithm for localization is based on the RSSI-based distance estimation. The choice for RSSI-based distance estimation is that it is more accurate compared to range-free techniques and carries no cost in terms of computation. The RSSI method estimates the distance among the two sensor nodes on the basis of the strength of radio signal.

The hybrid algorithm based on trilateration is used for the calculation of position. These two methods combined to improve the accuracy. Trilateration is the localization method that calculates the position based on distances from three references to a common point. A circle is drawn surrounded each reference point and the estimated position is determined by the intersection of the circles as shown in the Figure 2. Trilateration is based on the principle that any given occasion is subjected to specific radio frequency characteristics as this process is based on the received signal strength of radio signal between transmitter and receiver. In this method, there is a set up phase in which a grid is defined and the RSSI information is manually calculated and stored in the database.

In normal operations, a node entering the area will sense a signal received by the anchor nodes, which will then be compared with the predefined database. The position is estimated based on the closest match with the database. In our network, the position is calculated via the sink node. The main reason behind this is to keep the mobile node functional only without adding any computational cost in order to save as much energy as possible. The instantaneous RSSI samples may have random variations due to noise, interference, shadowing and more. The time-average of samples is carried out to filter the variations. Then, the mode of the filtered samples is taken to estimate distance as it most likely represents the line of sight (LoS) path. Distance estimation and the position computation are the two phases of this scheme.

\subsection{Distance Estimation}

RSSI is used to find the distance between target nodes in a given set of fixed node also known as anchor nodes. This distance can be estimated from RSSI by using log normal shadowing model:

Figure 2. Position calculation based on three references to a common point

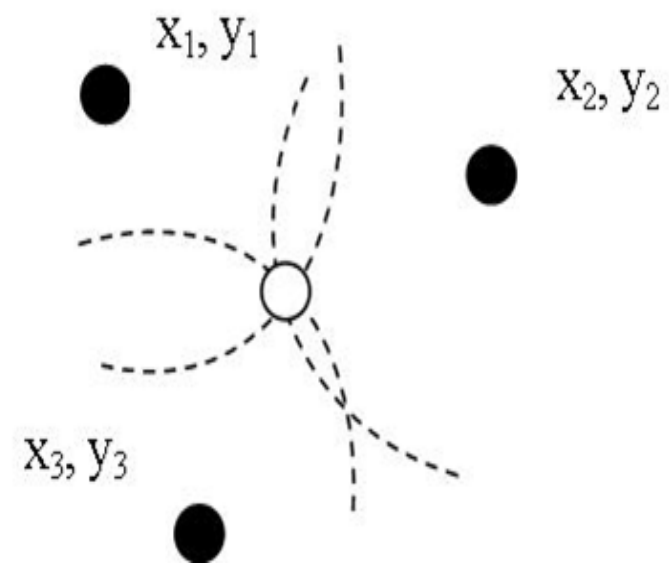




$$
R S S I(d)=-10 n \log _{10}(d)-C
$$

where, $R S S I$ is given as the function of distance, $n$ is path loss exponent, $d$ is the distance from transmitter and $C$ is environment constant. For the indoor environment, $n$ can range from 0 to 5 . Due to the exponential relationship, the accuracy of $n$ is critical to distance estimation.

\subsection{Position Computation}

In a given location of anchor nodes that are $\left(x_{1}, y_{1}\right),\left(x_{2}, y_{2}\right)$ and $\left(x_{3}, y_{3}\right)$.and the distances from the target to the anchors $\left(d_{1}, d_{2}\right.$ and $\left.d_{3}\right)$, we need to estimate the location of the target $\left(x_{0}, y_{0}\right)$. When the distance is larger between the two terminals, the received power is low. With the help of this received signal strength, we can find the distances between the transmitter and receiver. If the distance between the two terminals is known, we can find the location by using more than two terminals. In Figure 3, three receivers are shown. The target is transmitting and each receiver gets the different signal strength indication because each receiver is at a different distance from the transmitter:

$$
\left(x_{1}-x\right)^{2}+\left(y_{1}+y\right)^{2}=d_{1}^{2}
$$

$\left(x_{2}-x\right)^{2}+\left(y_{2}+y\right)^{2}=d_{2}^{2}$

$$
\left(x_{3}-x\right)^{2}+\left(y_{3}+y\right)^{2}=d_{3}^{2}
$$

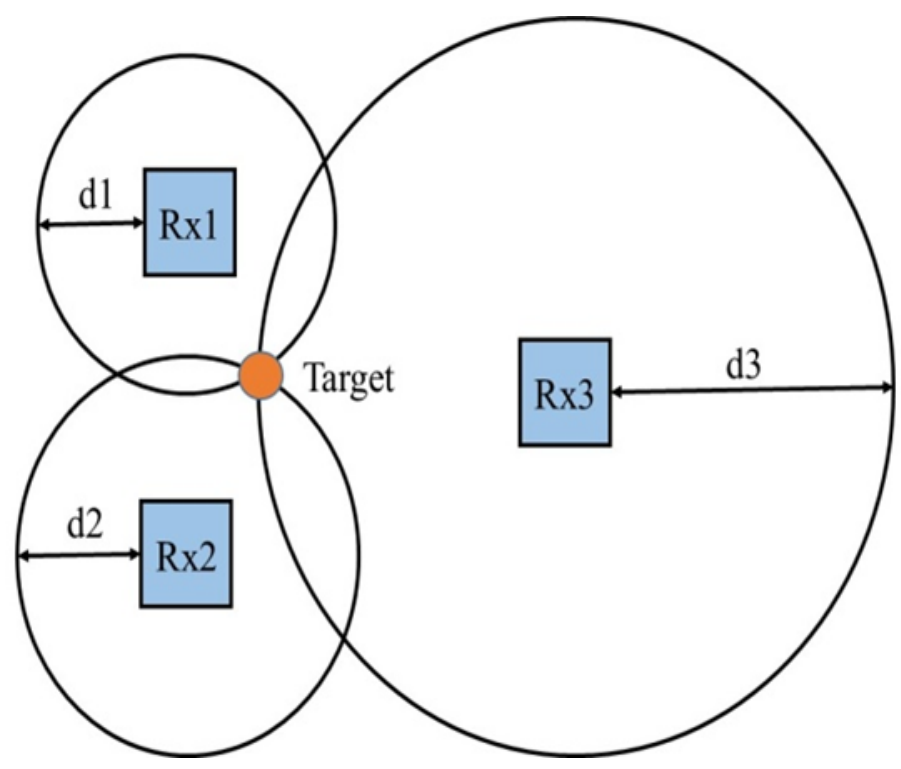


Equation 2, 3 and 4 are the formula for circles that are generated. Each circle being the radius that equals to the distance between the target and that particular receiver. The point where these three circles intersect is the location of target. Here, we can also find the actual geometric coordinates.

\section{IMPLEMENTATION}

The network topology used is mesh by placing the anchor nodes at the corner. The sensor node broadcasts message to the anchor node, which collects the series of RSSI samples. Then, the localization algorithm is implemented to estimate the distance of unknown sensor nodes in a network. For the network implementation, a 100x100 m grid matrix is used with four anchor nodes at the corner. As the anchor nodes are located within the each other's range, the anchor nodes shown in black color communicates directly with the sink node shown in green color. However, in the larger network deployment communication from anchor node to sink node, it will follow a multi-hop path in accordance with the mesh topology.

To create the framework for the localization of sensor node, we used Matlab version 8.1. The anchor node receives a message from an unknown node in the network and therefore gets the RSSI of the unknown node. Then, all of the anchor nodes transmit the message containing RSSI to the sink node as shown in Figure 4. After gathering all the messages, the sink node merges them into a single message. The algorithm then performs all the distance and localization calculation. For the implementation of the algorithm, the first phase is the experimental phase to determine how to convert the signal strength to the actual distance. Distance estimation is the starting point for the trilateration, after which the database is created. The anchor nodes do the data collection. The localization algorithm interfaces the sink node to estimate the distance based on RSSI. The distance estimation is done by the log-distance RSSI model.

The Localization algorithm is responsible for the data processing. For the trilateration calculation, only the three strongest signals from the four anchor nodes are used. In equation 2, 3 and $4,\left(x_{1}, y_{1}\right)$,

Figure 4. The Implementation of the network for Location Estimation

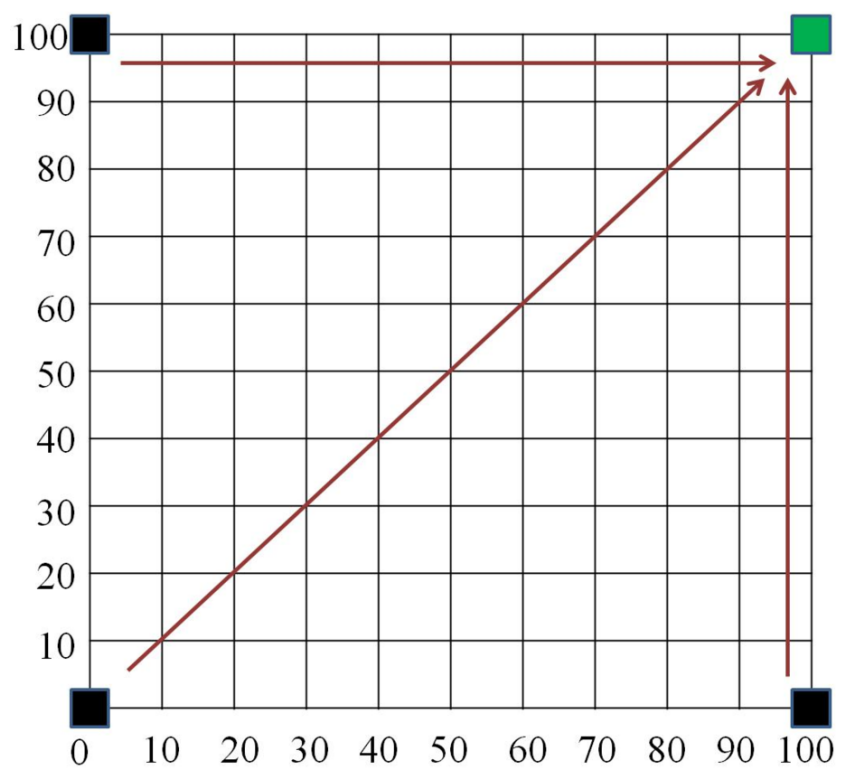

Anchor Node $\square$ Sink Node 
$\left(x_{2}, y_{2}\right)$ and $\left(x_{3}, y_{3}\right)$ are the coordinates of three anchor nodes whereas $\left(d_{1}, d_{2}\right.$ and $\left.d_{3}\right)$ are the respective distances to the unknown nodes. This can be simplified into a linear equation system that can be solved in the form of $P x=Q$, resulting in less computation. The final position estimation is an average of all the trilateration results:

$$
\begin{aligned}
& P=2\left[\begin{array}{ll}
x_{3}-x_{1} & y_{3}-y_{1} \\
x_{3}-x_{2} & y_{3}-y_{2}
\end{array}\right] \\
& Q=\left[\begin{array}{l}
\left(d_{1}^{2}-d_{3}^{2}\right)-\left(x_{1}^{2}-x_{3}^{2}\right)-\left(y_{1}^{2}-y_{3}^{2}\right) \\
\left(d_{2}^{2}-d_{3}^{2}\right)-\left(x_{2}^{2}-x_{3}^{2}\right)-\left(y_{2}^{2}-y_{3}^{2}\right)
\end{array}\right]
\end{aligned}
$$

\subsection{Sample Visualization and Test Phase}

To check how the algorithm works, we place some unknown sensor nodes at coordinates $(7,15),(16$, $57)$ and $(40,63)$ marked as positive (+) sign in blue color. By applying the trilateration localization, the nodes are approximately located at coordinates $(8,15),(18,57)$ and $(38,62)$ marked as circle $(0)$ sign in red color as shown in Figure 5.

The final estimation with the localization algorithm is approximately nearer to the true location of mobile sensors shown as red mark in the above figure. To test the algorithm, we have placed mobile sensor nodes at different locations within the $100 * 100 \mathrm{~m}$ grid. The accuracy of the actual and estimated position is discussed in the experimental analysis section.

Figure 5. Test phase deployment of 3 sensor nodes and their location estimation

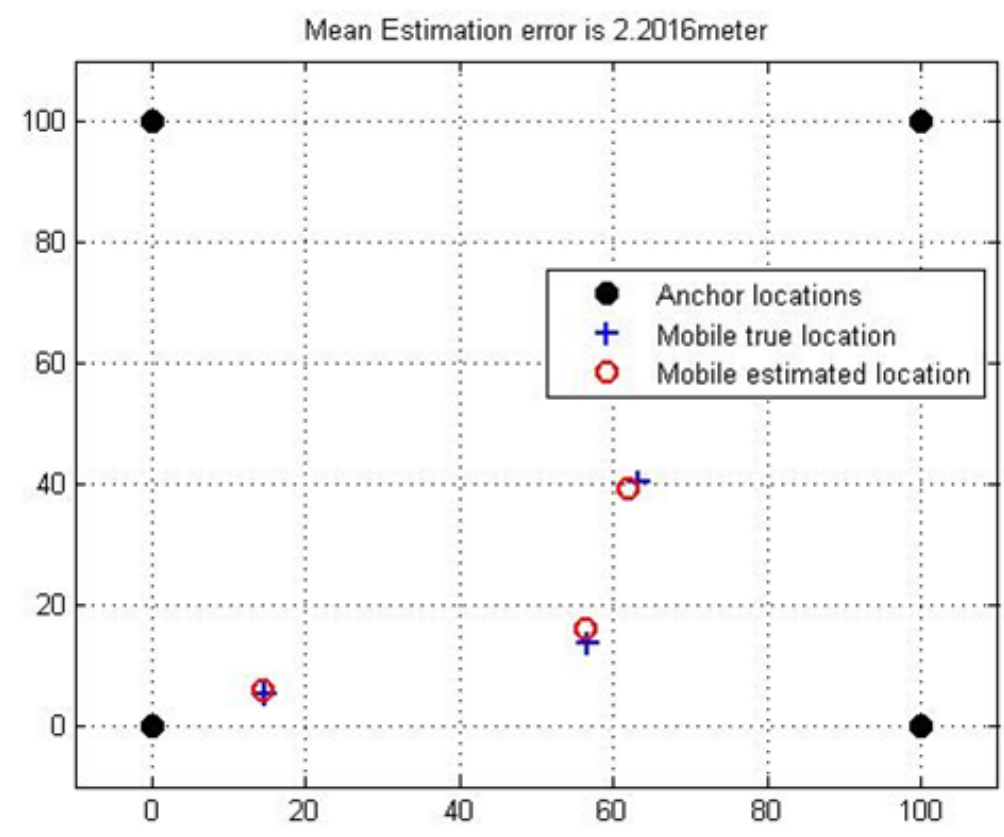




\section{EXPERIMENTAL ANALYSIS}

To check the performance of the localization algorithm vis-à-vis the deployment of sensors, three cases to evaluate the estimation accuracy for localization are conducted.

\subsection{Case 1}

The process of execution is done via Matlab 8.1. Figure 6 shows the deployment of mobile node over the area. In case 1, the mobile nodes are taken as 50, anchor nodes are taken as 4 and the network size is taken as 100 . In the figure, black dots represent the location of anchor nodes, the blue positive $(+)$ sign represents the true location of mobile nodes, and red circle represents the estimated location of mobile nodes.

In case 1, the 50 mobile nodes are plotted over the network size 100, keeping the anchor node 4 as constant. Table 1 represents the distance between the mobile nodes and the anchor nodes over the network size 100. Here, the anchor nodes are 4 and the mobile nodes are 50. The table represents the distance only for 10 sensor nodes.

\subsection{Case 2}

Figure 7 shows the deployment of mobile node over the area. In case 2, the mobile nodes are taken as 100, anchor nodes remains constant as 4 and the network size is taken as 200. In this figure, black dots represent the location of anchor nodes, the blue positive (+) sign represents the true location of mobile nodes, and red circle represents the estimated location of mobile nodes.

In case 2, the 100 mobile nodes are plotted over the network size 200 keeping the anchor node 4 as constant.

Table 2 represents the distance between the mobile nodes and the anchor nodes over the network size 200. Here, the anchor nodes are 4 and the mobile nodes are 100.

\subsection{Case 3}

Figure 8 shows the deployment of mobile node over the area. In case 3, the mobile nodes are taken as 100 , anchor nodes remains constant as 4 and the network size is taken as 500. In this figure, black

Figure 6. Deployment of 50 mobile sensor nodes, with 4 anchor nodes in $100 * 100 \mathrm{~m}$ grid

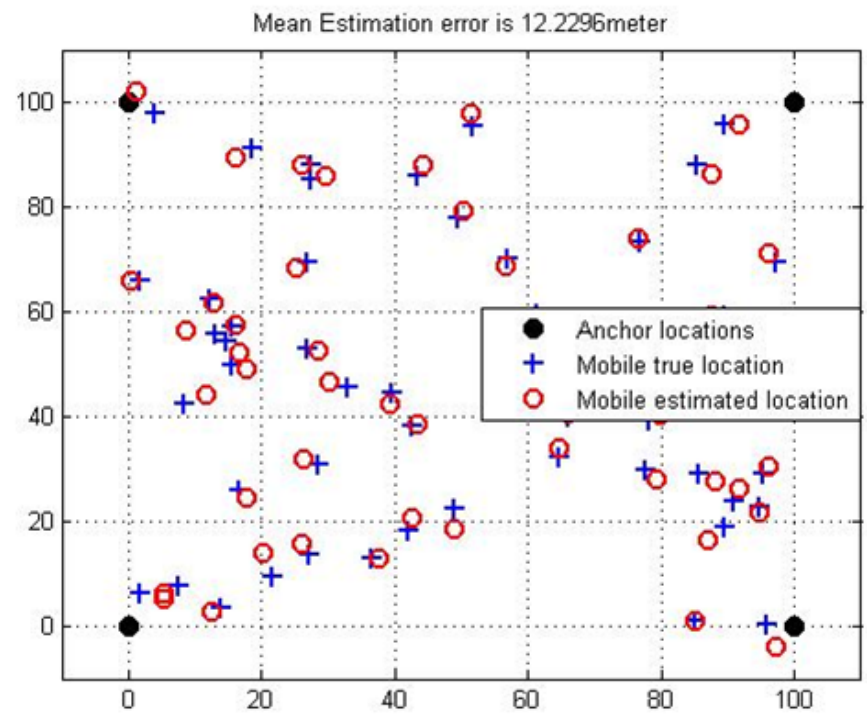


Table 1. Distance between all the nodes and anchor nodes in the network

\begin{tabular}{|l|c|c|c|c|c|c|c|c|c|c|}
\hline \multirow{2}{*}{ Anchor Nodes } & \multicolumn{10}{|c|}{ Sensor Nodes } \\
\cline { 2 - 13 }$y$ & $\mathbf{1}$ & $\mathbf{2}$ & $\mathbf{3}$ & $\mathbf{4}$ & $\mathbf{5}$ & $\mathbf{6}$ & $\mathbf{7}$ & $\mathbf{8}$ & $\mathbf{9}$ & $\mathbf{1 0}$ \\
\hline 1 & 21.69 & 126.9 & 114.64 & 49.85 & 91.60 & 26.00 & 93.15 & 66.27 & 97.26 & 87.66 \\
\hline 2 & 85.99 & 82.98 & 74.948 & 52.08 & 101.2 & 90.24 & 13.03 & 119.5 & 125.4 & 105.8 \\
\hline 3 & 86.07 & 97.68 & 91.45 & 102.50 & 44.48 & 78.31 & 128.60 & 33.72 & 19.18 & 37.33 \\
\hline 4 & 119.7 & 17.52 & 28.86 & 103.6 & 61.94 & 116.6 & 89.61 & 105 & 81.48 & 70.09 \\
\hline
\end{tabular}

Figure 7. Deployment of 100 mobile sensor nodes, with 4 anchor nodes in $200 * 200 \mathrm{~m}$ grid

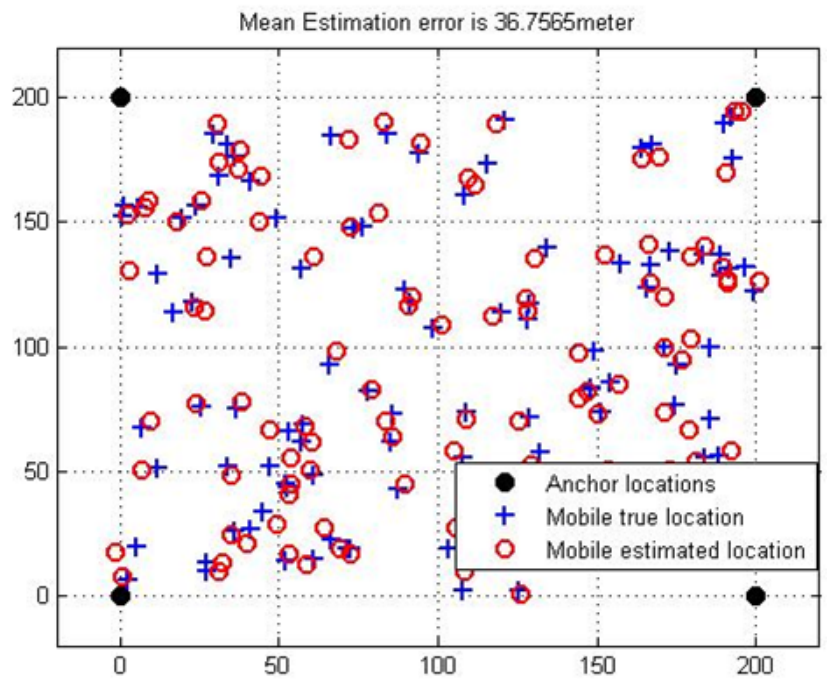

Table 2. Distance estimation among all nodes and anchor nodes in the network for case 2

\begin{tabular}{|l|c|c|c|c|c|c|c|c|c|c|}
\hline \multirow{3}{*}{ Anchor Nodes } & \multicolumn{10}{|c|}{ Sensor Nodes } \\
\cline { 2 - 13 } & $\mathbf{1}$ & $\mathbf{2}$ & $\mathbf{3}$ & $\mathbf{4}$ & $\mathbf{5}$ & $\mathbf{6}$ & $\mathbf{7}$ & $\mathbf{8}$ & $\mathbf{9}$ & $\mathbf{1 0}$ \\
\hline 1 & 268.3 & 6.81 & 184.3 & 168 & 212.9 & 225.9 & 105.2 & 233.1 & 169.6 & 141.9 \\
\hline 2 & 189.8 & 198 & 246 & 88.47 & 136.6 & 206.3 & 98.23 & 137.5 & 97.38 & 63.82 \\
\hline 3 & 190.2 & 193.5 & 38.45 & 196.8 & 179.9 & 121.5 & 208.6 & 198.7 & 189.1 & 226.3 \\
\hline 4 & 14.50 & 276.8 & 167.4 & 135.4 & 75.23 & 79.37 & 205.2 & 63.96 & 128.4 & 187.4 \\
\hline
\end{tabular}

dots represent the location of anchor nodes, the blue positive (+) sign represents the true location of mobile nodes, and red circle represents the estimated location of mobile nodes. In case 3, the 100 mobile nodes are plotted over the network size 500 keeping the anchor node 4 as constant.

Table 3 represents the distance between the mobile nodes and the anchor nodes over the network size 500. Here, the anchor nodes are 4 and the mobile nodes are 100 . We have evaluated the performance of UNL algorithm for the localization accuracy by Matlab version 8.1. We also compared the proposed algorithm with existing algorithm in the literature on DV-Hop (23) and APIT 
Figure 8. Deployment of 100 mobile sensor nodes, with 4 anchor nodes in $500 * 500 \mathrm{~m}$ grid

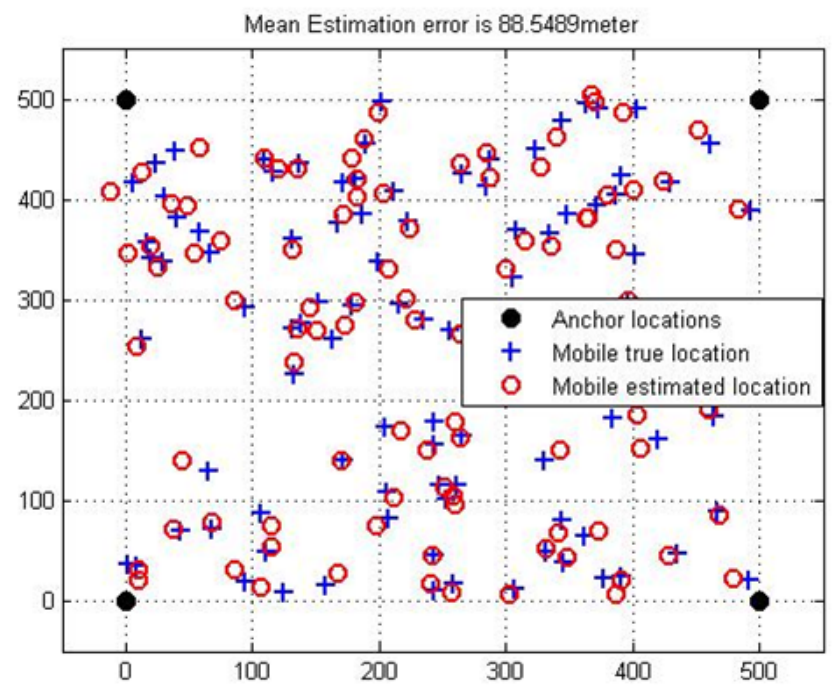

Table 3. Distance between all the nodes and anchor nodes in the network

\begin{tabular}{|c|c|c|c|c|c|c|c|c|c|c|}
\hline \multirow{2}{*}{ Anchor Nodes } & \multicolumn{10}{|c|}{ Sensor Nodes } \\
\hline & 1 & 2 & 3 & 4 & 5 & 6 & 7 & 8 & 9 & 10 \\
\hline 1 & 309.7 & 458.4 & 390.9 & 440 & 339.2 & 437.8 & 312.7 & 462.8 & 382 & 474.7 \\
\hline 2 & 455.0 & 528.4 & 112.4 & 469.8 & 580 & 646.3 & 287 & 284.2 & 297.7 & 95.89 \\
\hline 3 & 262.8 & 197.3 & 615.1 & 253.3 & 164.5 & 67.21 & 427 & 452.7 & 411 & 621 \\
\hline 4 & 424.4 & 328.6 & 488 & 302.2 & 498.4 & 480.1 & 408.5 & 267.5 & 334.1 & 411.6 \\
\hline
\end{tabular}

(15). The simulated results are obtained from average trials and we found that the localization error is comparatively less than the existing algorithms of localization.

\section{CONCLUSION}

In conclusion there are large numbers of parameters that must be measured for better quality estimation of distance. RSSI, or received signal strength indicator, provides the best solution for the localization problem in WSNs. Radio frequency, transmission power, orientation of nodes and the localization algorithms are the parameter that we have analyzed in our experiments affecting the estimation of distance.

On the basis of these analyses, we have proposed an algorithm for the estimation of distance and localization. The transmission power is the most important factor for efficient distance estimation. The algorithm provides better level of accuracy for the estimated positions and is directly related with the number of the anchor nodes that are deployed in the sensor network. Increasing the number of anchor nodes can lead to higher accuracy of the position estimation. The range-based approaches provide much better accuracy in comparison with range-free approaches. The range-based approaches require extra hardware with the sensor nodes for the measurement of different parameters, which thereby increases the size, weight, energy and cost of the network. 
The proposed algorithm estimates the position of the unknown node via the minimal connectivity and the information of proximity in a network. Therefore this approach does not require any extra hardware, hence saving the energy, weight and size costs. The experimental result of the proposed algorithm presents the localization accuracy without compromising complexity and cost. The algorithm can be implemented for different wireless platform for locating the nodes accurately. The system is having its limitation for medical implant because of its limited radio channel of human body. The propagation of radio signal in human body is difficult to predict. The time based positioning is an alternate solution and it requires homogenous propagation of radio signal. The future work can be extended by considering its application for the real time positioning of wireless implantation. Furthermore, the experiments can be extended by providing security to the system by utilizing some security algorithms along with the unknown node localization algorithm. These security algorithms can enhance the level of security and also we can achieve better energy computation. 


\section{REFERENCES}

Alasiry, A. H., \& Ohyama, S. (2012). Range-based localization with area verification for sparse distributed wireless sensor networks. In 2012 International Conference on Advanced Computer Science and Information Systems (ICACSIS) (pp. 43-46). IEEE.

Ali, K., Neogy, S., \& Das, P. K. (2010). Optimal energy-based clustering with gps-enabled sensor nodes. In 2010 Fourth International Conference on Sensor Technologies and Applications (pp. 13-18). IEEE. doi:10.1109/ SENSORCOMM.2010.103

Bianchi, V., Ciampolini, P., \& De Munari, I. (2018). RSSI-based indoor localization and identification for ZigBee wireless sensor networks in smart homes. IEEE Transactions on Instrumentation and Measurement, 68(2), 566-575. doi:10.1109/TIM.2018.2851675

Cao, J. (2011). A new hybrid algorithm on TDOA localization in wireless sensor network. In 2011 IEEE International Conference on Information and Automation (pp. 606-610). IEEE.

Chen, X., He, J., \& Lei, B. (2011). An Improved Algorithm of Sensors Localization in Wireless Sensor Network. In International Conference on Computer Science, Environment, Ecoinformatics, and Education (pp. 572-578). Springer. doi:10.1007/978-3-642-23324-1_92

Gu, S., Yue, Y., Maple, C., Wu, C., \& Liu, B. (2013). Challenges in mobile localisation in wireless sensor networks for disaster scenarios. In 2013 19th International Conference on Automation and Computing (pp. 1-6). IEEE.

Han, G., Jiang, J., Zhang, C., Duong, T. Q., Guizani, M., \& Karagiannidis, G. K. (2016). A survey on mobile anchor node assisted localization in wireless sensor networks. IEEE Communications Surveys and Tutorials, 18(3), 2220-2243. doi:10.1109/COMST.2016.2544751

Haque, I. T., \& Assi, C. (2013). Profiling-based indoor localization schemes. IEEE Systems Journal, 9(1), 76-85. doi:10.1109/JSYST.2013.2281257

Khan, H., Hayat, M. N., \& Rehman, Z. U. (2017). Wireless sensor networks free-range base localization schemes: A comprehensive survey. In 2017 International Conference on Communication, Computing and Digital Systems (C-CODE) (pp. 144-147). IEEE. doi:10.1109/C-CODE.2017.7918918

Khan, U. A., Kar, S., \& Moura, J. M. (2009). Distributed sensor localization in random environments using minimal number of anchor nodes. IEEE Transactions on Signal Processing, 57(5), 2000-2016. doi:10.1109/ TSP.2009.2014812

Kim, H., \& Han, S. W. (2014). An efficient sensor deployment scheme for large-scale wireless sensor networks. IEEE Communications Letters, 19(1), 98-101. doi:10.1109/LCOMM.2014.2372015

Luan, J., Zhang, R., Zhang, B., \& Cui, L. (2014). An improved monte carlo localization algorithm for mobile wireless sensor networks. In 2014 Seventh International Symposium on Computational Intelligence and Design (Vol. 1, pp. 477-480). IEEE. doi:10.1109/ISCID.2014.217

Ma, D., Er, M. J., Wang, B., \& Lim, H. B. (2012). Range-free wireless sensor networks localization based on hop-count quantization. Telecommunication Systems, 50(3), 199-213. doi:10.1007/s11235-010-9395-y

Maddumabandara, A., Leung, H., \& Liu, M. (2015). Experimental evaluation of indoor localization using wireless sensor networks. IEEE Sensors Journal, 15(9), 5228-5237. doi:10.1109/JSEN.2015.2438193

Marta, M., Yang, Y., \& Cardei, M. (2009). Energy-efficient composite event detection in wireless sensor networks. In International Conference on Wireless Algorithms, Systems, and Applications (pp. 94-103). Springer. doi:10.1007/978-3-642-03417-6_10

Patwari, N., Ash, J. N., Kyperountas, S., Hero, A. O., Moses, R. L., \& Correal, N. S. (2005). Locating the nodes: Cooperative localization in wireless sensor networks. IEEE Signal Processing Magazine, 22(4), 54-69. doi:10.1109/MSP.2005.1458287

Phoemphon, S., So-In, C., \& Nguyen, T. G. (2018). An enhanced wireless sensor network localization scheme for radio irregularity models using hybrid fuzzy deep extreme learning machines. Wireless Networks, 24(3), 799-819. doi:10.1007/s11276-016-1372-2 
Shahzad, F., Sheltami, T. R., \& Shakshuki, E. M. (2016). Multi-objective optimization for a reliable localization scheme in wireless sensor networks. Journal of Communications and Networks (Seoul), 18(5), 796-805. doi:10.1109/JCN.2016.000108

Shi, Y., Cui, Q., Cao, S., Zhang, X., \& Tao, X. (2014). Performance relationship between distributed and centralised cooperative localisations. Electronics Letters, 50(2), 127-128. doi:10.1049/el.2013.3452

Stojkoska, B., Ivanoska, I., \& Davcev, D. (2009). Wireless sensor networks localization methods: Multidimensional scaling vs. semidefinite programming approach. In International Conference on ICT Innovations (pp. 145-155). Springer.

Wan, X., Shen, L., Chen, Z., \& Xu, H. (2018). An efficient virtual nodes-based APIT localization algorithm with low computational cost. In 2018 IEEE 23rd International Conference on Digital Signal Processing (DSP) (pp. 1-4). IEEE. doi:10.1109/ICDSP.2018.8631829

Yoon, S., Soysal, O., Demirbas, M., \& Qiao, C. (2011). Coordinated locomotion and monitoring using autonomous mobile sensor nodes. IEEE Transactions on Parallel and Distributed Systems, 22(10), 1742-1756. doi:10.1109/ TPDS.2011.133

Yuan, Y., Huo, L., Wang, Z., \& Hogrefe, D. (2018). Secure APIT localization scheme against sybil attacks in distributed wireless sensor networks. IEEE Access: Practical Innovations, Open Solutions, 6, 27629-27636. doi:10.1109/ACCESS.2018.2836898

Zeng, F., Li, W., \& Guo, X. (2018). An Improved DV-Hop Localization Algorithm Based on Average Hop and Node Distance Optimization. In 2018 2nd IEEE Advanced Information Management, Communicates, Electronic and Automation Control Conference (IMCEC) (pp. 1336-1339). IEEE. doi:10.1109/IMCEC.2018.8469655

Zhang, B., \& Yu, F. (2008). An energy efficient localization algorithm for wireless sensor networks using a mobile anchor node. In 2008 International Conference on Information and Automation (pp. 215-219). IEEE. doi:10.1109/ICINFA.2008.4607999

Zhao, X., Zhang, X., Sun, Z., \& Wang, P. (2018). New wireless sensor network localization algorithm for outdoor adventure. IEEE Access: Practical Innovations, Open Solutions, 6, 13191-13199. doi:10.1109/ ACCESS.2018.2813082

\footnotetext{
Pradeep Kumar Singh is currently working as a Professor and Head in the department of CSE at ABES Engineering College, Ghaziabad, Uttar Pradesh, India.. He is having 9 years of vast experience in academics at reputed colleges and university of India. He has completed his Ph.D. in Computer Science \& Engineering from Gautam Buddha University (State Government University), Greater Noida, UP, India. He received his M. Tech. (CSE) with Distinction from Guru Gobind Singh Indraprastha University, New Delhi, India. He has obtained his B. Tech. (CSE) from Uttar Pradesh Technical University (UPTU), Lucknow, India. Dr. Singh is having life membership of Computer Society of India (CSI). He is member of ACM, IACSIT-Singapore and IAENG-Hong Kong. He has worked as publicity chair of two IEEE Conferences and reviewer of various Journals. He is Associate Editor of International Journal of Information Security and Cybercrime a scientific peer reviewed journal from Romania.
} 